\title{
Link between spermine oxidase and apoptosis antagonizing transcription factor: A new pathway in neuroblastoma
}

\author{
EMILIANO FRATINI ${ }^{1}$, MANUELA CERVELLI ${ }^{1,2}$, PAOLO MARIOTTINI $^{1,2}$, YUTA KANAMORI $^{3}$, \\ ROBERTO AMENDOLA ${ }^{4}$ and ENZO AGOSTINELLI ${ }^{2,3}$ \\ ${ }^{1}$ Department of Science, 'Roma Tre’ University, I-00146 Rome; \\ ${ }^{2}$ International Polyamines Foundation - ONLUS, I-00159 Rome; \\ ${ }^{3}$ Department of Biochemical Sciences ‘A. Rossi Fanelli', 'La Sapienza' University, I-00185 Rome; \\ ${ }^{4}$ ENEA, UT Bio-Rad RAB, I-00123 Rome, Italy
}

Received June 4, 2019; Accepted July 19, 2019

DOI: $10.3892 /$ ijo.2019.4878

\begin{abstract}
Neuroblastoma (NB) is a heterogeneous extra-cranial childhood type of cancer, responsible for approximately $15 \%$ of all paediatric cancer-related deaths. Although several critical genetic aberrations have been related to NB, only a few established molecular markers have been associated with prognosis [V-myc avian myelocytomatosis viral oncogene $(M Y C N)$ locus amplification, deletions of part of chromosome 1p, 11q23 and gain of 17q]. Regrettably, direct evidence of NB-related tumour suppressors or oncogenes has not been currently identified at these chromosomal regions. MYCN locus amplification is present in approximately 20-30\% of cases and is associated with a poor clinical outcome, representing the most important genetic prognostic marker. The functional guidelines for the prognosis of NB identify high-risk patients ( $<40 \%$ survival probabilities), but fail to identify patients at low and intermediate stages of the disease, which remains an issue to be resolved in NB. It has been shown
\end{abstract}

Correspondence to: Professor Enzo Agostinelli, Department of Biochemical Sciences 'A. Rossi Fanelli', 'La Sapienza' University, I-00185 Rome, Italy

E-mail: enzo.agostinelli@uniroma1.it

Abbreviations: AATF, apoptosis-antagonizing-factor; APE1, apurinic/apyrimidinic endonuclease $1 ; \mathrm{H}_{2}$ DCFDA, 2',7'-dichlorodihydrofluorescein diacetate; FCM, flow cytometry; PAOX, peroxisomal $\mathrm{N}^{1}$-acetyl-spermine/spermidine oxidase; NAC, $N$-acetyl-cysteine; $\quad$ MDL-72527, $\quad \mathrm{N}, \mathrm{N}^{1}$-Bis(2,3-butadienyl)-1,4butanediamine; NB, neuroblastoma; ODC, ornithine decarboxylase; PA, polyamines; PI, propidium iodide; PUT, putrescine; SPD, spermidine; SSAT, spermidine/spermine N1-acetyltransferase; SMOX, spermine oxidase; SPM, spermine; MYCN, V-myc avian myelocytomatosis viral oncogene, neuroblastoma-derived homolog; TdT, 3'-terminal deoxy-transferase

Key words: spermine oxidase, apoptosis antagonizing transcription factor, neuroblastoma, reactive oxygen species that in NB cell lines and in a total-spermine oxidase (SMOX) transgenic mouse model, SMOX overexpression induces cellular stress via reactive oxygen species (ROS) imbalance. In this study, we demonstrated that the high expression level of the cytoprotective gene, apoptosis-antagonizing transcription factor $(A A T F)$, was driven by $S M O X$ gene overexpression in both NB cells and Total-SMOX mice. The anti-apoptotic effect of $A A T F$ was supported by analysing the inhibition of the expression of the pro-apoptotic genes, $B A X, B A K$ and $P U M A$, which were decreased, in both the in vitro and in vivo SMOX overexpressing model systems investigated. On the whole, this study supports the hypothesis that the SMOX gene can be considered as a novel anti-apoptotic marker in NB.

\section{Introduction}

Neuroblastoma (NB) is the most frequent extra-cranial childhood type of cancer and is responsible for approximately $15 \%$ of all paediatric deaths due to cancer $(1,2)$. NB is substantially heterogeneous $(3,4)$. Several critical genetic aberrations have been associated with NB; however, few molecular markers have been associated with the prognosis of the disease. Among these, the V-myc avian myelocytomatosis viral oncogene neuroblastoma derived homolog (MYCN) locus amplification is the most important, being present in approximately $20-30 \%$ of all NB cases, and is strongly associated with a poor prognosis (5). Deletions of part of $1 \mathrm{p}$, of 11q23 $(6,7)$ and the gain of 17q chromosomes (8) has also been shown to be associated with to a negative outcome. However, NB-related tumour suppressors or oncogenes have not been identified at these chromosomal regions. Functional guidelines for the prognosis of patients with NB were drawn as risk grouping. The Children's Oncology Group (COG) (9) incorporated the tumour stage as assessed by the International NB Staging System (INSS), MYCN amplification level, and histology according to Shimada et al (10). These guidelines identify high-risk patients, with disseminated disease, frequent $M Y C N$ amplification and poorly differentiated tumour cells. The survival probability is $<40 \%$, regardless of the cancer treatment being administered. In recent years, gene expression profiles and NB outcome have been studied 
with different technical platforms and analytical methods in order to distinguish high-risk from low-risk tumours; these methods however, result in poor prognostic relevance when they are used to discriminate patients with low-and intermediate-stage disease $(11,12)$, an issue that has now become a major clinical challenge in the field of NB. Oxidative stress plays an important role in switching from cell proliferation to cell death (13), and the metabolic pathways responsible for the polyamine (PA) inter-conversion and degradation are responsible for oxidant by-products $(14,15)$. Over the past years, spermine (SPM) metabolism has been found to be closely related to DNA oxidation in NB cell lines; however, the ectopic expression of spermine oxidase (SMOX), that specifically oxidizes SPM to produce spermidine (SPD), 3 -aminopropanal and hydrogen-peroxide $(16,17)$, induces DNA damage without any increase in cell mortality (18). SMOX over-activity provokes sub-lethal chronic DNA damage and repair, visualized as an induction of apurinic/apyrimidinic endonuclease protein (APE1) and the hyper-phosphorylation of the $\mathrm{H} 2 \mathrm{AX}$ histone, a well characterized marker of DNA damage and repair (19). In the present study, we further characterized the effects of SMOX, stably transfected into NB cells. In parallel, we investigated the effects of SMOX in a mouse model (Total-SMOX mice) overexpressing SMOX in all tissues $(20,21)$, and also examined the expression of the cyto-protective gene, apoptosis-antagonizing transcription factor (AATF) (otherwise known as Che-1) (22). AATF has been characterized to be stress-activated and to inhibit apoptosis, and it is induced and stabilized upon DNA damage $(22,23)$. In this study, it was demonstrated that a high level of AATF expression is associated with SMOX gene overexpression in both NB cells and in Total-SMOX mice. Of note, the anti-apoptotic effect due to AATF has recently been described as a transcriptional competitor in the promoter regions of the p53-driven expression of the pro-apoptotic genes $B A X, B A K$ and PUMA $(24,25)$. The results presented in this study also confirm the antagonizing role of AATF towards BAX, BAK and PUMA in the in vitro and in vivo SMOX-overexpressing model systems investigated.

\section{Materials and methods}

Cells, cell culture and reagents. All reagents were purchased from Sigma-Aldrich, unless otherwise specified. The inhibitor of FAD-dependent SMOX activity, N,N'-Bis(2,3-butadienyl)-1,4-butanediamine dihydrochloride (MDL-72527, herein referred to as MDL), was a gift from Hoechst Marion Roussel Inc. Plastic-wares were purchased from Nunc (Nunc A/S). The transfections and grow th conditions of the parental N18TG2 murine NB cell line (Sigma-Aldrich), pcDNA3-transfected (NP), pcDNA3-SMOX-transfected (NS) and pcDNA3- $\gamma$ SMO-transfected $(\mathrm{NG})$ cell lines were as previously described in the study by Cervelli et al (26), Amendola et al (18) and Bianchi et al (19). The $\gamma \mathrm{SMO}$ isoform is a splicing variant of SMOX lacking the FAD domain (26). The cells were pre-treated with MDL at a final concentration of $50 \mu \mathrm{M}$ for $24 \mathrm{~h}$. For ROS counteraction, the cells were pre-treated with $\mathrm{N}$-acetyl-cysteine (NAC) at a final concentration of $10 \mathrm{mM}$ of $24 \mathrm{~h}$. The control cells were cells treated in the absence of MDL and NAC.
Animals. A total of 9 syngeneic (SG) male mice and 9 Total-SMOX transgenic (TG) male mice (3 months old, with a mean body weight of $30 \mathrm{~g}$ ), were used as the experimental subjects. In the experiments, only male mice were used in order to exclude all biochemical and behavioural changes due to female cycle hormones. They were born and raised at the 'Stazione per la Tecnologia Animale' at the University of Tor Vergata and they were transferred to the animal house of the University of Roma Tre for the experiments. The animals were housed in an environment with a controlled temperature $\left(20 \pm 1^{\circ} \mathrm{C}\right)$, humidity $(55 \pm 10 \%)$ and a 12 -h light/dark cycle. The housing conditions were in accordance with standard laboratory settings including cage enrichment with free access to food and water.

Transgenic GFP-SMOX (former JoSMOX) mice described in the study by Cervelli et al (27) were crossed with Total-CRE mice to obtain the Total-SMOX genetic line described in the study by Ceci et al (20). In order to stabilize the Total-SMOX transgenic line, mice were further genetically stabilised backcrossing 10 times with C57BL/6 mice (20). The genotyping for the Total-SMOX mice was carried out using the $\beta$-gal staining of the tail biopsies from the mouse pups and confirmed by PCR methods according to Ceci et al (20). In the experiments, as control mice, the syngeneic littermates were used. All experiments were performed on independent groups of mice. The organs (brain, intestine and heart) were obtained from sacrificed SG and TG mice by cervical dislocation. The experiments were carried out in accordance with the ethical guidelines for the conduct of animal research of the European Community's Council Directive 77/499/EEC e 81/309/ EEC. Formal approval of these experiments was obtained from the Italian Ministry of Health (Official Italian Regulation D.L.vo 26/2014, 'Authorization from Ministero della Salute no. 964/2015-PR'). All experiments were performed on independent groups of mice. All efforts were made to minimize the number of animals used and their suffering.

$R N A$ isolation, reverse transcription-quantitative $P C R$ (RT-qPCR) and semi-quantitative RT-PCR. Total RNA from the NG,NP, NS cell lines, and from the SG and TG mouse brains, intestines and hearts was isolated using the GeneElute system, and reverse transcribed into cDNA using the SuperScript First-Strand Synthesis System (Invitrogen; Thermo Fisher Scientific), according to the manufacturer's instructions. For quantitative PCR (qPCR), cDNA was amplified by SYBR Premix Ex Taq (Takara Bio Inc.) according to the manufacturer's instructions. Specific primers pairs were designed using Primer Express Software (Applied Biosystems). The thermocycling conditions were as follows: $95^{\circ} / 30 \mathrm{sec}$, followed by 40 cycles of $\left[95^{\circ} / 5 \mathrm{sec}\right.$; $60-63^{\circ}$ (according to Table SI) $\left./ 30 \mathrm{sec}\right]$.

Three experimental replicates were performed, and the results were analysed using the relative expression software tool (REST) (28). The sequence and position of the specific primers for $A A T F, B A K, B A X$ and $P U M A$ are listed in Table SI. The sequence and position of the specific primers for SMOX, $\beta$-actin and RPS7 as the housekeeping reference genes have been described elsewhere $(18,19,27)$. For semi-quantitative RT-PCR, the Takara PCR Amplification kit was used (Takara Bio Inc.). The thermocycling conditions were as follows: $94 \% \mathrm{~min}$, followed by 30 cycles of 
[94\% $/ 1 \mathrm{~min} ; 60-63^{\circ}$ (according to Table $\left.\mathrm{SI}\right) / 30 \mathrm{sec} ; 72^{\circ} / 30 \mathrm{sec}$ ] and then $72 \% \mathrm{~min}$. The amplified products were run on a $1.5 \%$ agarose gel. Gel images using ethidium bromide were obtained with automatic exposure by Diana III dedicated device (Raytest Italia).

Analysis of ROS levels, stress and apoptosis. The levels of intracellular hydrogen peroxide were determined by flow cytometric (FCM) analysis of the fluorescence intensity of 2',7'-dichlorodihydrofluorescein diacetate (H2DCFDA) (Invitrogen; Thermo Fisher Scientific). Briefly, the cells were treated with H2DCFDA for 30 min with/without pre-treatment for $24 \mathrm{~h}$ with $10 \mathrm{mM}$ NAC. At least $2 \times 10^{4}$ cells were analyzed by a FACSCalibur flow cytometer (BD Biosciences), with laser excitation set at 495 and a $525 \mathrm{~nm}$ emission filter to detect green fluorescence. Oxidative stress, on the basis of number of 8-oxo-dG residues (29), was analysed using the OxyDNA kit, following the manufacturer's instructions (Merck KGaA). Negative control was obtained by omitting the fluorescence probe-mix from the reaction. The levels of $\gamma \mathrm{H} 2 \mathrm{AX}$ accumulation were determined by FCM analysis of the green fluorescence intensity, only inside the G1 sub-cohort of cells evidenced by DNA red fluorescence using $1 \mu \mathrm{g} / \mathrm{ml}$ of propidium iodide (PI), with laser excitation set at 488 and $630 \mathrm{~nm}$ band pass emission filter to detect both red and green fluorescence (30). The cells were stained with a primary $\gamma \mathrm{H} 2 \mathrm{AX}$, rabbit polyclonal antibody (4418-APC-020; Trevigen) at a dilution of $1: 100$, overnight a $4^{\circ} \mathrm{C}$, and then with a secondary monoclonal anti-rabbit FITC-conjugated antibody (sc-2359; Santa Cruz Biotechnology) at a dilution of 1:200, for $2 \mathrm{~h}$ at room temperature. The level of 3'-terminal deoxy-transferase (TdT) by the TUNEL method was used to detect apoptosis, following the manufacturer's instructions (in situ Cell Death Fluorescent kit; Roche Diagnostic S.p.A.) and was performed as previously described (31). AATF was detected and quantified by both semi-quantitative RT-PCR (see above) and western blot analysis. Protein extraction and western blot analysis were carried out as previously described by Ceci et al (20). The Briefly, tissues were homogenized in $5 \mathrm{vol}(\mathrm{w} / \mathrm{v})$ of ice-cold lysis buffer (50 mM Tris-HCl pH 7.5, 10\% glycerol, $320 \mathrm{mM}$ sucrose, $1 \%$ Triton, $1 \mathrm{mM}$ PMSF, $1 \mathrm{X}$ complete protease inhibitor cocktail) using a Potter Elvehjem Tissue Homogenizer for $45 \mathrm{sec}$. The samples were kept on ice for $30 \mathrm{~min}$ and then centrifuged at $16,100 \mathrm{x}$ g for $15 \mathrm{~min}$ at $4^{\circ} \mathrm{C}$. The supernatant was collected and protein determination was carried out as previously described by Ceci et al (20). Primary rabbit polyclonal antibody developed against AATF (dilution 1:1,000, not commercially available; provided by Professor Fanciulli, Università degli Studi di Milano-Bicocca) (22) was used for western blot analysis (incubated overnight at $4^{\circ} \mathrm{C}$ ). Equal proteins amounts (20 $\mu \mathrm{g} / \mathrm{sample})$ were loaded on electrophoresis in a $10 \%$ polyacrylamide gel and transferred onto nitrocellulose membranes. The membranes were blocked with $5 \%$ non-fat dry milk for $1 \mathrm{~h}$ and incubated overnight at $4{ }^{\circ} \mathrm{C}$ with a polyclonal anti- $\beta$-actin (dilution 1:2,000, cat. no. A5060; Sigma-Aldrich S.r.l) diluted in $2.5 \%$ non-fat dry milk. $\beta$-actin was taken as the reference. Secondary peroxidase-labelled anti-rabbit IgG antibody (dilution 1:5,000, cat. no. SA00001-2, in $1.25 \%$ non-fat dry milk) was from Proteintech. The secondary antibody was incubated for $1 \mathrm{~h}$ at room temperature. Detection was performed using ECL Western blotting detection reagents (GE Healthcare).
Densitometric measurements were obtained using ImageJ 1.52a software (Wayne Rasband National Institutes of Health).

Analysis of enzymatic activities of PA metabolism. PA oxidases [SMOX and the peroxisomal FAD-dependent enzyme $\mathrm{N}^{1}$-acetyltransferase oxidase (PAOX)] activity was assayed as previously described $(19,32,33)$. Ornithine decarboxylase (ODC) and spermidine/spermine $\mathrm{N}^{1}$-acetyltransferase (SSAT) activities were determined using ${ }^{14} \mathrm{C}$-labelled substrate and scintillation counting of end metabolized products, as described elsewhere $(18,19)$. Cell pellets were sonicated on ice and frozen in aliquots. The protein concentration was determined using the Bradford method and at least 3 determination replicas were carried out for the enzymatic activity of SMOX, PAOX, ODC and SSAT.

Statistical analysis. Significant differences at $\mathrm{P}<0.05$ were evaluated by one-way ANOVA, followed by the multiple comparison Tukey post-hoc test (SPSS-11 statistical dedicated software; SPSS Inc.). All experiments were repeated 3 times unless otherwise indicated.

\section{Results}

SMOX induces cellular stress via an imbalance in ROS generation, but not apoptosis. In previous studies, SMOX activity has been described to induce a chronic sub-lethal DNA damage, with a 3 -fold increase in 8-oxo-dG residues, but with no increase in cell mortality being observed $(18,19,31)$. Treatments with inhibitors of PA oxidases have been shown to abolish the higher amount of oxidative stress in different cell line models $(34,35)$. Similar results were obtained in the present study, since treatment with NAC was able to counteract ROS overproduction (36). ROS (Fig. 1A, left panel) and 8-oxo-dG (Fig. 1B, left panel) were induced by SMOX in the NS cells, as shown by the FCM histograms of H2DCFDA and 8-oxo-dG residues, when compared with both NP and NG cells. A final concentration of $10 \mathrm{mM}$ NAC treatment scavenged ROS overproduction (Fig. 1A, right panel) and hampered 8-oxo-dG residues (Fig. 1B, right panel) in NS cells. The $\gamma \mathrm{H} 2 \mathrm{AX}$ phosphorylated content was also evaluated to monitor the onset of cellular response to DNA damage (Fig. 1C, left panel). SMOX induced $\gamma \mathrm{H} 2 \mathrm{AX}$ phosphorylation in the NS cells and NAC subverted this event (Fig. 1C, right panel). These data confirm the ability of SMOX to produce oxidative cellular stress, driving cells to a preliminary phase of repair. Notably, we confirm previous data on NB cells $(18,19)$ and in human colon adenocarcinoma cell (31), whereby SMOX overexpression does not induce cell mortality and apoptosis per se when evaluated by the ipo-G0/G1 sub-population and TUNEL assay, as shown in Fig. S1.

SMOX induces AATF gene expression via an imbalance in ROS generation. The cellular ROS disequilibrium can drive cells to enter a complex mechanistic molecular pathway to arrest the cell cycle and promote DNA repair or apoptosis. From this point of view, SMOX represents an apparently self-contradictory response, since it has the ability to induce preliminary stress without driving the onset of apoptosis. Thus, we wished to examine the level of the AATF gene (22). Upon 
A

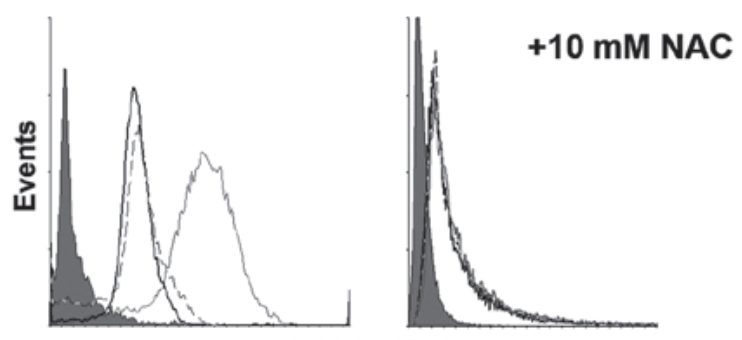

H2DCF-FLH1

B

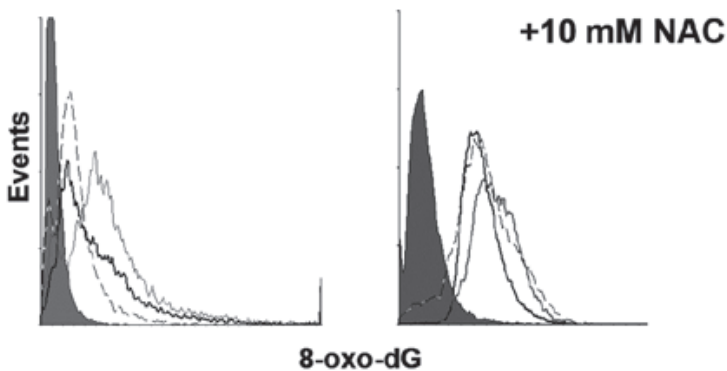

C

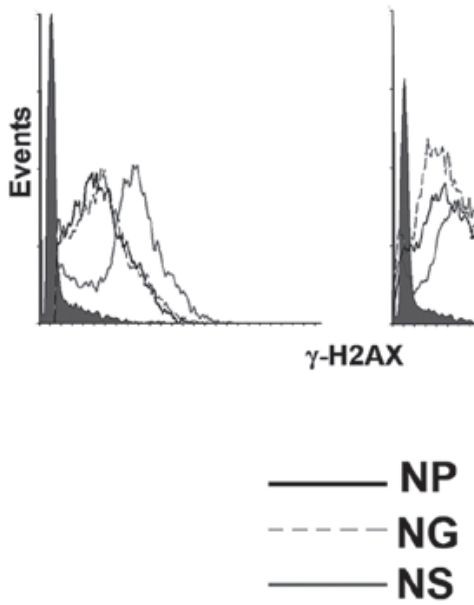

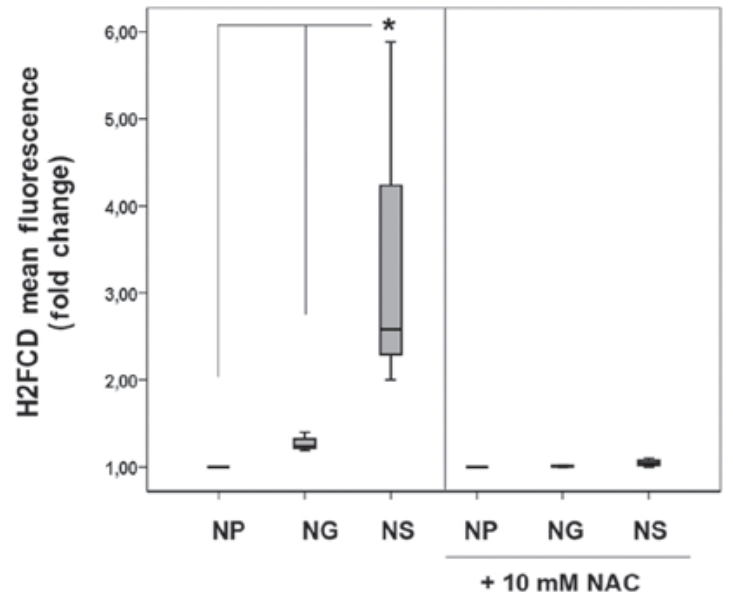
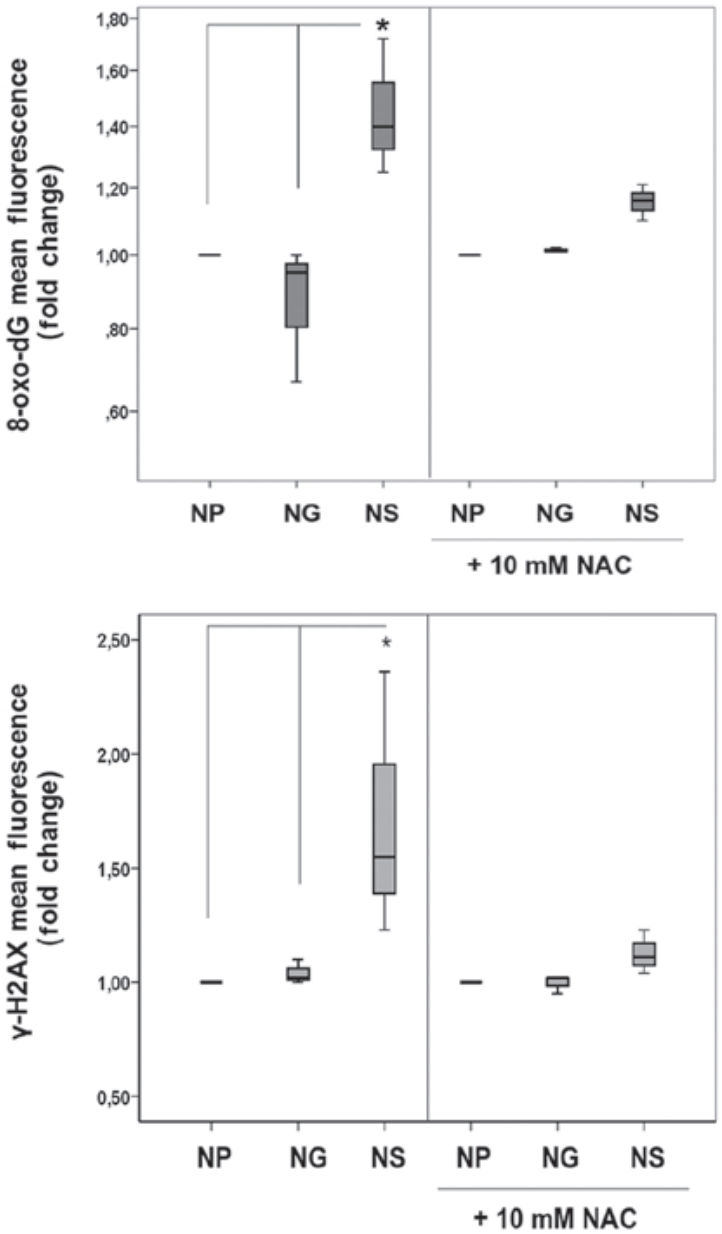

Figure 1. SMOX induces oxidative stress. Mono-parametric FCM analysis of (A) H2DCF, (B) 8-oxo-dG, and (C) $\gamma \mathrm{H} 2 \mathrm{AX}$. Auto-fluorescence is closed to the y-axes (dark grey-filled histograms). NP, N18TG2 cells transfected with pcDNA3 (black line); NG, N18TG2 cells transfected with pcDNA3/ $\gamma$ SMO (dashed line); NS, N18TG2 cells transfected with pcDNA3/SMOX. In the right panels, analyses were performed following pre-treatment of the cells for $24 \mathrm{~h}$ with $10 \mathrm{mM}$ of NAC as a ROS scavenger. H2DCF and 8-oxo-dG are gathered as FLH-1 green fluorescence emission. $\gamma \mathrm{H} 2 \mathrm{AX}$ is quantified within the Go/G1 cellular sub-population (PI counter-stain) to avoid the S-phase $\gamma \mathrm{H} 2 \mathrm{AX}$-positive cells as a confounding factor. In the left panels, histograms are representative of at least 20,000 events gated. In the right panels, the Whisker plots represent the statistical analysis of the fold change of the mean and the dispersion of the fluorescence in the cell population, relative to NP cells. Data were analysed by one-way ANOVA, followed by Tukey's post hoc test. "P<0.05 vs. NP cells and NG cells. SMOX, spermine oxidase; NAC, $N$-acetyl-cysteine.

genotoxic stress, AATF has been described to become phosphorylated and to subsequently translocate to the nucleus to bind the PUMA, BAX and $B A K$ promoter regions (23). PUMA, $B A X$ and $B A K$ are well known pro-apoptotic genes driven by p53, and NB cells are almost exclusively p53-proficient (23).
AATF mRNA expression was evaluated by semi-quantitative RT-PCR in the NP, NG and NS cells, under normal growth conditions and following treatment with NAC (Fig. 2A). At 25 cycles, AATF mRNA expression was higher in the NS cells. With the increasing PCR cycles, amplification rises to saturate 


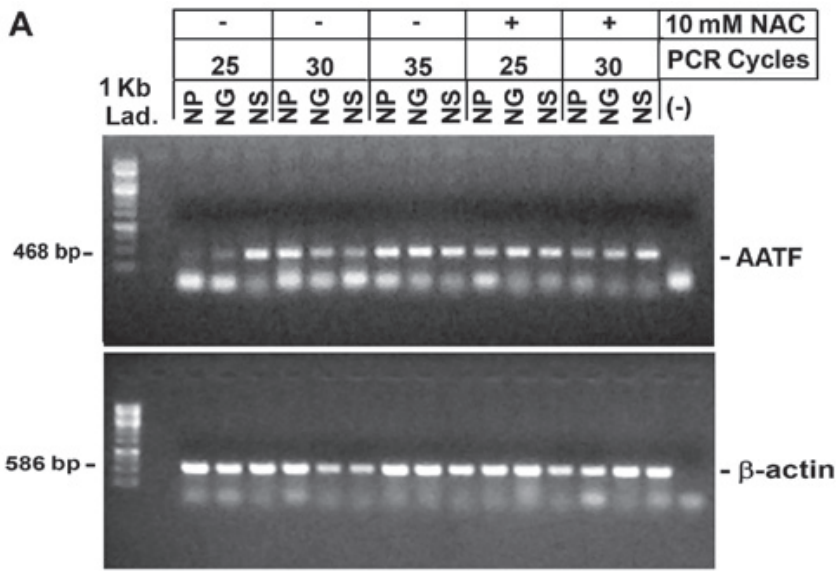

B

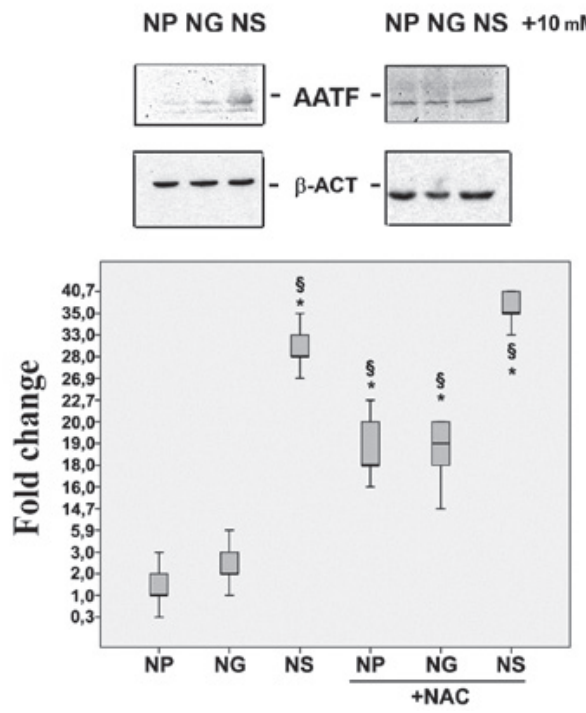

Figure 2. SMOX induces AATF expression. (A) Semi-quantitative RT-PCR quantification of the antagonizing apoptosis transcription factor (AATF) and $\beta$-actin expression levels in NP, NS, NG cells, in the presence $10 \mathrm{mM}$ NAC as a ROS scavenger at different PCR cell cycles (as indicated). The symbol '-' represents the negative control of the PCR reaction. (B) Western blot analysis of AATF in NP, NG and NS cells in the presence of $10 \mathrm{mM}$ NAC as a ROS scavenger. The Whisker plot represents the fold changes in AATF protein content vs. NP, calculated as arbitrary densitometric units normalized vs. actin signals of 3 independent experiments. Data were analysed by one-way ANOVA, followed by Tukey's post hoc test. "P $<0.05$ vs. NP cells; ${ }^{\circledR} \mathrm{P}<0.05$ vs. NG cells. SMOX, spermine oxidase; AATF, apoptosis antagonizing-factor; $\beta$-ACT, $\beta$-actin; NAC, $N$-acetyl-cysteine; NP cells. NP, N18TG2 cells transfected with pcDNA3; NS, N18TG2 cells transfected with pcDNA3/SMOX; NG, N18TG2 cells transfected with pcDNA3/ $\gamma \mathrm{SMO}$

the signals. The exhaustive ROS deprivation due to treatment with $10 \mathrm{mM}$ NAC (Fig. 2A), enforced AATF expression in all the cell lines. AATF protein accumulation detected by western blot analysis was in full accordance with the RNA expression data (Fig. 2B, left panel), while NAC treatment induced the over-accumulation of AATF protein in all cell lines (Fig. 2B, right panel). As a first step, a semi-quantitative RT-PCR indicated the diminished amount of mRNA for the pro-apoptotic genes, BAK, BAX and PUMA, in the NS cells (Fig. S2A). MDL treatment induced an equal expression of these pro-apoptotic genes in the NP, NG and NS cell lines (Fig. S2A). The expression levels of BAK, BAX and PUMA were then quantified by qPCR, as a ratio with the RPS7 housekeeping reference gene, without analysing the NAC-treated cells, since they
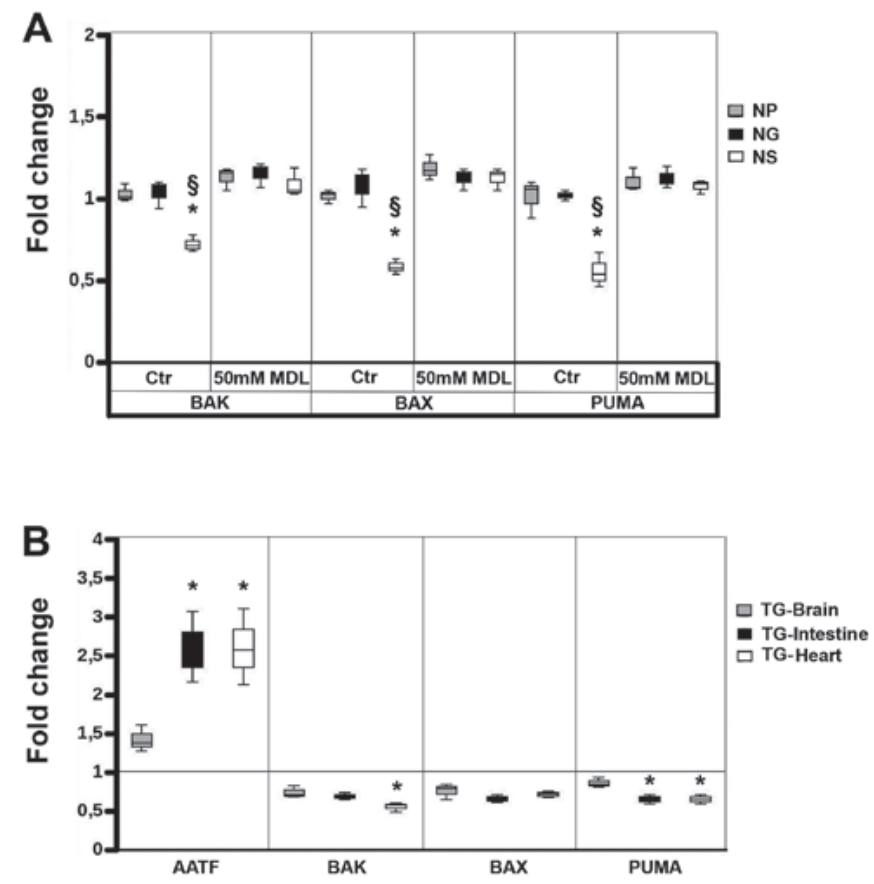

Figure 3. SMOX indirectly inhibits the expression of the pro-apoptotic genes, BAK, BAX and PUMA. (A) Fold change of BAK, BAX and PUMA expression between NG (black bars) and NS (white bars) cell lines vs. NP (black bars). Whisker-plot graph of qPCR normalized vs.RPS7 as the control housekeeping gene, in the absence or presence of $50 \mu \mathrm{M}$ MDL as a SMOX inhibitor. (B) Fold change of AATF, BAK, BAX and PUMA expression between tissues from Total-SMOX transgenic mice (TG) vs. syngeneic mice. Whisker-plot graph of qPCR normalized vs. RPS7 as the control housekeeping gene. TG-Brain (grey bars), TG-Intestine (black bars), and TG-Heart (white bars). Data were analysed by one-way ANOVA, followed by Tukey's post hoc test. "P>0.05 vs. NP cells in (A) and TG-Brain in (B); ${ }^{{ }^{\$} \mathrm{P}}<0.05$ vs. NG cells in (A). SMOX, spermine oxidase, AATF, apoptosis antagonizing-factor, BAK, Bcl-2 antagonist/killer, BAX, Bcl-2-associated $\mathrm{X}$ protein, PUMA, p53 upregulated modulator of apoptosis; MDL-72527, $\mathrm{N}, \mathrm{N}$-Bis(2,3-butadienyl)-1,4-butanediamine dihydrochloride; NP cells. NP, N18TG2 cells transfected with pcDNA3; NS, N18TG2 cells transfected with pcDNA3/SMOX; NG, N18TG2 cells transfected with pcDNA3/ $\gamma$ SMO.

exhibited an equal amount of AATF gene transcripts. The results of qPCR confirmed the lower mRNA expression levels of BAK, BAX and PUMA in the NS vs. the NP and NG cell lines, and the normalization effect due to MDL (Fig. 3A). Subsequently, we analysed these effects of SMOX overexpression in tissues from the genetic-engineered conditional mouse model, Total-SMOX (20). When comparing the level of AATF in different organs between the transgenic and syngeneic animals, we observed a higher level of this anti-apoptotic factor in the intestinal tract and heart tissues than in the brain, according to SMOX expression in these organs (Fig. 3B). Consistent with the results obtained in vitro, the AATF levels were associated with SMOX overexpression, as demonstrated by semi-quantitative RT-PCR (Fig. S2B) and quantified by qPCR (Fig. 3B), where the expression of genes are reported as ratio between syngeneic vs. transgenic mice. AATF activation was associated with the transcriptional inhibition of $B A K, B A X$ and PUMA, thus representing indirect evidence in vivo of the anti-apoptotic activity of SMOX. Taken together, these results strongly indicate that SMOX may act as an indirect inhibitor of apoptosis, via an imbalance in ROS generation, and may be considered a tumorigenic gene. 

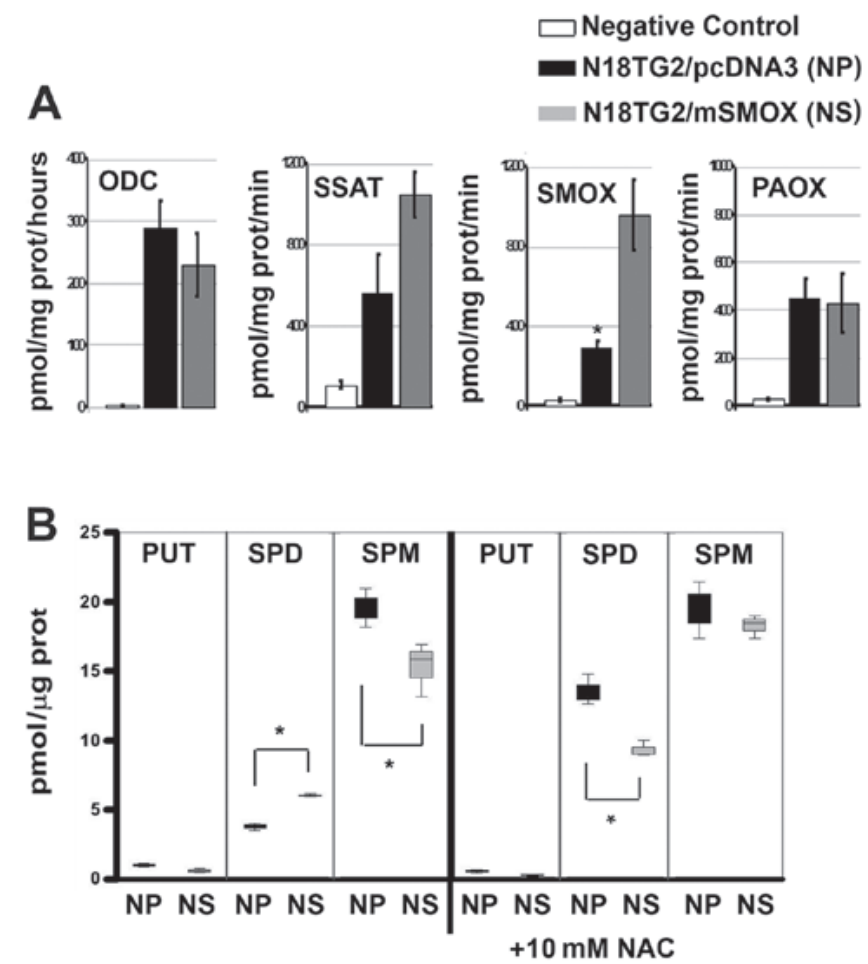

Figure 4. PA metabolism. (A) Column bar graphs of ODC, SSAT, SMOX and PAOX activities in expressed as $\mathrm{pmol} / \mathrm{mg}$ proteins/h in NP and NS cells. White columns represent the negative controls of enzymatic reactions, with no cellular extracts. "P $>0.05$, difference in SMOX activity between NP and NS cell lines. (B) Column bar graphs of PUT, SPD and SPM concentrations expressed as pmoles $/ \mu \mathrm{g}$ of protein, in NP and NS cells and in the presence of $10 \mathrm{mM}$ NAC (as indicated). PUT, SPD, and SPM concentrations obtained from NG control cells were identical to NP cells, and then omitted for the sake of clarity. Data were analysed by one-way ANOVA, followed by Tukey's post hoc test. "P>0.05 vs. NP cells. NP, N18TG2 cells transfected with pcDNA3 (black bars); NS, N18TG2 cells transfected with pcDNA3/SMOX (grey bars). PA, polyamine; ODC, ornithine decarboxylase; SSAT, spermidine/spermine $\mathrm{N}^{1}$-acetyltransferase; SMOX, spermine oxidase; APAO, $\mathrm{N}^{1}$-acetylpolyamine oxidase; PUT, putrescine; SPD, spermidine; SPM, spermine.

SMOX and PA metabolism in NB cells. We also found that PA metabolism in the NS cells was consistent with what was expected $(19,37)$. Namely, a low ODC activity corresponds to a higher SSAT activity and to a 3- or 4-fold enhancement in SMOX activity (19,31) (Fig. 4A). The intracellular PA concentrations followed the enzymatic variation consistently, with less putrescine (PUT) and more SPD, mainly due to SMOX overactivity, although the amount of SPM was consistently buffered by the interconversion of the PA metabolism (Fig. 4B, whisker box-plot). Of note, NAC induced an increase in the level of intracellular SPD in both NP and NS cell lines.

\section{Discussion}

Previous studies have demonstrated that SMOX activity is involved in the delivery of oxidative stress $(18,19,35)$. At such a level, ROS may act as signalling molecules in various intracellular processes $(38,39)$, one of these being apoptosis. However, without any further induction of damage, SMOX did not induce the onset of apoptosis (18,19,35 and this study). Recently, the $A A T F$ gene has been characterized to be stress-activated and to inhibit apoptosis $(22,23)$. AATF is induced and stabilized upon DNA damage $(22,23)$ and, in this study, its expression was found to be associated with SMOX overexpression in $\mathrm{NB}$ cells and tissues. In our in vitro and in vivo experimental models, the expression levels of BAK, BAX and PUMA were downregulated in the presence of SMOX overexpression and the SMOX-induced increase in the AATF levels. However, the chromosomal gains on the AATF locus (17q12) with the consistent augmented mRNA expression level of AATF are associated per se with a negative outcome in NB patients (23), thus justifying that SMOX activity can preserve cell survival under stress conditions, playing an indirect anti-apoptotic role, inducing AATF expression. This could have detrimental effects on cancer prognosis $(40,41)$. The PA bio-synthesis rates are associated with a negative prognosis (37), and each biosynthetic and catabolic step of PA metabolism is a putative target for cancer diagnosis. The findings of this study confirm that dysfunctions in PA metabolism are strictly related to cancer. In conclusion, ROS overproduction due to SMOX activity may activate AATF and may indirectly inhibit the apoptosis of NB cells.

\section{Acknowledgements}

The authors would like to thank the 'International Polyamine Foundation - ONLUS' for the availability to look up the polyamines documentation. The authors are indebted to Professor Roberto Fanciulli (Università degli Studi di Milano-Bicocca) for providing the antibodies against AATF.

\section{Funding}

This study was funded by the generous support of 'La Sapienza' University of Rome and the Italian MIUR (Ministero dell'Istruzione, dell'Università e della Ricerca). This study was also supported by the Italian Ministry of Education, University and Research (MIUR), Grant to Department of Science, University 'Roma Tre'-'Dipartimenti di Eccellenza', (Legge 232/2016, Articolo 1, Comma 314-337) and by the 'Roma Tre' University of Rome contribution to the laboratories (CAL/2017 and CAL/2018) to MC. PM and EA would also like to thank Wakunaga Pharmaceutical Co., Ltd. for the scholarship given to $\mathrm{YK}$ for supporting his $\mathrm{PhD}$ research.

\section{Availability of data and materials}

All data generated or analysed during this study are included in this published article or are available from the corresponding author on reasonable request.

\section{Authors' contributions}

EF, MC, PM, YK, EA and RA conceived this study and coordinated the collaboration among the authors. EF, RA and MC performed all the experiments. EF, MC, EA and RA optimized the protocols for the analyses. All authors wrote the manuscript and all authors have read and approved the final manuscript.

\section{Ethics approval and consent to participate}

The experiments were carried out in accordance with the ethical guidelines for the conduct of animal research of the European 
Community's Council Directive 77/499/EEC e 81/309/EEC. Formal approval of these experiments was obtained from the Italian Ministry of Health (Official Italian Regulation D.L.vo 26/2014, "Authorization from Ministero della Salute no. 964/2015-PR"). All experiments were performed on independent groups of mice. All efforts were made to minimize the number of animals used and their suffering.

\section{Patient consent for publication}

Not applicable.

\section{Competing interests}

The authors declare that they have no competing interests.

\section{References}

1. Brodeur GM and Maris JM: Neuroblastoma. In: Principles and Practice of Pediatric Oncology. Pizzo PA and Poplack DG (eds). JB Lippincott, Philadelphia, pp933-970, 2006.

2. Steliarova-Foucher E, Colombet M, Ries LAG, Moreno F, Dolya A, Bray F, Hesseling P, Shin HY, Stiller CA, Bouzbid S, et al; IICC-3 contributors: International incidence of childhood cancer, 2001-10: A population-based registry study. Lancet Oncol 18: 719-731, 2017.

3. Brodeur GM: Neuroblastoma: Biological insights into a clinical enigma. Nat Rev Cancer 3: 203-216, 2003.

4. Ngan ES: Heterogeneity of neuroblastoma. Oncoscience 2: 837-838, 2015.

5. Maris JM, Hogarty MD, Bagatell R and Cohn SL: Neuroblastoma. Lancet 369: 2106-2120, 2007.

6. Attiyeh EF, London WB, Mossé YP, Wang Q, Winter C, Khazi D, McGrady PW, Seeger RC, Look AT, Shimada H, et al; Children's Oncology Group: Chromosome 1p and 11q deletions and outcome in neuroblastoma. N Engl J Med 353: 2243-2253, 2005.

7. Mlakar V, Jurkovic Mlakar S, Lopez G, Maris JM, Ansari M and Gumy-Pause F: 11q deletion in neuroblastoma: A review of biological and clinical implications. Mol Cancer 16: 114, 2017.

8. Bown N, Cotterill S, Lastowska M, O'Neill S, Pearson AD, Plantaz D, Meddeb M, Danglot G, Brinkschmidt C, Christiansen H, et al: Gain of chromosome arm 17q and adverse outcome in patients with neuroblastoma. N Engl J Med 340: 1954-1961, 1999

9. O'Leary M, Krailo M, Anderson JR and Reaman GH; Children's Oncology Group: Progress in childhood cancer: 50 years of research collaboration, a report from the Children's Oncology Group. Semin Oncol 35: 484-493, 2008.

10. Shimada H, Chatten J, Newton WA Jr, Sachs N, Hamoudi AB, Chiba T, Marsden HB and Misugi K: Histopathologic prognostic factors in neuroblastic tumors: Definition of subtypes of ganglioneuroblastoma and an age-linked classification of neuroblastomas. J Natl Cancer Inst 73: 405-416, 1984.

11. Oberthuer A, Berthold F, Warnat P, Hero B, Kahlert Y, Spitz R, Ernestus K, König R, Haas S, Eils R, et al: Customized oligonucleotide microarray gene expression-based classification of neuroblastoma patients outperforms current clinical risk stratification. J Clin Oncol 24: 5070-5078, 2006.

12. Wang Q, Diskin S, Rappaport E, Attiyeh E, Mosse Y, Shue D, Seiser E, Jagannathan J, Shusterman S, Bansal M, et al: Integrative genomics identifies distinct molecular classes of neuroblastoma and shows that multiple genes are targeted by regional alterations in DNA copy number. Cancer Res 66: 6050-6062, 2006.

13. Schumacker PT: Reactive oxygen species in cancer cells: Live by the sword, die by the sword. Cancer Cell 10: 175-176, 2006.

14. Casero RA Jr and Marton LJ: Targeting polyamine metabolism and function in cancer and other hyperproliferative diseases. Nat Rev Drug Discov 6: 373-390, 2007.

15. Cervelli M, Angelucci E, Germani F, Amendola R and Mariottini P: Inflammation, carcinogenesis and neurodegeneration studies in transgenic animal models for polyamine research. Amino Acids 46: 521-530, 2014
16. Cervelli M, Amendola R, Polticelli F and Mariottini P: Spermine oxidase: Ten years after. Amino Acids 42: 441-450, 2012.

17. Polticelli F, Salvi D, Mariottini P, Amendola R and Cervelli M: Molecular evolution of the polyamine oxidase gene family in Metazoa. BMC Evol Biol 12: 90, 2012.

18. Amendola R, Bellini A, Cervelli M, Degan P, Marcocci L, Martini F and Mariottini P: Direct oxidative DNA damage, apoptosis and radio sensitivity by spermine oxidase activities in mouse neuroblastoma cells. Biochim Biophys Acta 1755: 15-24, 2005.

19. Bianchi M, Bellini A, Cervelli M, Degan P, Marcocci L, Martini F, Scatteia M, Mariottini P and Amendola R: Chronic sub-lethal oxidative stress by spermine oxidase overactivity induces continuous DNA repair and hypersensitivity to radiation exposure. Biochim Biophys Acta 1773: 774-783, 2007.

20. Ceci R, Duranti G, Leonetti A, Pietropaoli S, Spinozzi F, Marcocci L, Amendola R, Cecconi F, Sabatini S, Mariottini P, et al: Adaptive responses of heart and skeletal muscle to spermine oxidase overexpression: Evaluation of a new transgenic mouse model. Free Radic Biol Med 103: 216-225, 2017.

21. Cervelli M, Leonetti A, Duranti G, Sabatini S, Ceci R and Mariottini P: Skeletal muscle pathophysiology: The emerging role of spermine oxidase and spermidine. Med Sci (Basel) 6: 6, 2018.

22. Bruno T, De Nicola F, Iezzi S, Lecis D, D'Angelo C, Di Padova M, Corbi N, Dimiziani L, Zannini L, Jekimovs C, et al: Che-1 phosphorylation by ATM/ATR and Chk2 kinases activates p53 transcription and the G2/M checkpoint. Cancer Cell 10: 473-486, 2006.

23. Liu X, Cai S, Zhang C, Liu Z, Luo J, Xing B and Du X: Deacetylation of NAT10 by Sirt1 promotes the transition from rRNA biogenesis to autophagy upon energy stress. Nucleic Acids Res 46: 9601-9616, 2018.

24. Höpker K, Hagmann H, Khurshid S, Chen S, Hasskamp P, Seeger-Nukpezah T, Schilberg K, Heukamp L, Lamkemeyer T, Sos ML, et al: AATF/Che-1 acts as a phosphorylation-dependent molecular modulator to repress p53-driven apoptosis. EMBO J 31: 3961-3975, 2012

25. Sang Q, Liu X, Wang L, Qi L, Sun W, Wang W, Sun Y and Zhang H: Sun Y andZhang H: CircSNCA downregulation by pramipexole treatment mediates cell apoptosis and autophagy in Parkinson's disease by targeting miR-7. Aging (Albany NY) 10: 6, 2018.

26. Cervelli M, Bellini A, Bianchi M, Marcocci L, Nocera S, Polticelli F, Federico R, Amendola R and Mariottini P: Mouse spermine oxidase gene splice variants. Nuclear subcellular localization of a novel active isoform. Eur J Biochem 271: 760-770, 2004.

27. Cervelli M, Bellavia G, D'Amelio M, Cavallucci V, Moreno S, Berger J, Nardacci R, Marcoli M, Maura G, Piacentini M, et al: A new transgenic mouse model for studying the neurotoxicity of spermine oxidase dosage in the response to excitotoxic injury. PLoS One 8: e64810, 2013.

28. Pfaffl MW, Horgan GW and Dempfle L: Relative expression software tool (REST) for group-wise comparison and statistical analysis of relative expression results in real-time PCR. Nucleic Acids Res 30: e36, 2002.

29. Cadet J, Douki T, Gasparutto D and Ravanat JL: Oxidative damage to DNA: Formation, measurement and biochemical features. Mutat Res 531: 5-23, 2003.

30. Huang X, Okafuji M, Traganos F, Luther E, Holden E and Darzynkiewicz Z: Assessment of histone H2AX phosphorylation induced by DNA topoisomerase I and II inhibitors topotecan and mitoxantrone and by the DNA cross-linking agent cisplatin. Cytometry A 58: 99-110, 2004.

31. Amendola R, Cervelli M, Fratini E, Sallustio DE, Tempera G, Ueshima T, Mariottini P and Agostinelli E: Reactive oxygen species spermine metabolites generated from amine oxidases and radiation represent a therapeutic gain in cancer treatments. Int J Oncol 43: 813-820, 2013.

32. Cervelli M, Fratini E, Amendola R, Bianchi M, Signori E, Ferraro E, Lisi A, Federico R, Marcocci L and Mariottini P: Increased spermine oxidase (SMO) activity as a novel differentiation marker of myogenic $\mathrm{C} 2 \mathrm{C} 12$ cells. Int J Biochem Cell Biol 41: 934-944, 2009.

33. Cervelli M, Bellavia G, Fratini E, Amendola R, Polticelli F, Barba M, Federico R, Signore F, Gucciardo G, Grillo R, et al: Spermine oxidase (SMO) activity in breast tumor tissues and biochemical analysis of the anticancer spermine analogues BENSpm and CPENSpm. BMC Cancer 10: 555, 2010. 
34. Pledgie A, Huang Y, Hacker A, Zhang Z, Woster PM, Davidson NE and Casero RA Jr: Spermine oxidase SMO(PAOh1), Not $\mathrm{N}^{1}$-acetylpolyamine oxidase $\mathrm{PAO}$, is the primary source of cytotoxic $\mathrm{H}_{2} \mathrm{O}_{2}$ in polyamine analogue-treated human breast cancer cell lines. J Biol Chem 280: 39843-39851, 2005.

35. Capone C, Cervelli M, Angelucci E, Colasanti M, Macone A, Mariottini $\mathrm{P}$ and Persichini T: A role for spermine oxidase as a mediator of reactive oxygen species production in HIV-Tat-induced neuronal toxicity. Free Radic Biol Med 63: 99-107, 2013.

36. Sun SY: N-acetylcysteine, reactive oxygen species and beyond. Cancer Biol Ther 9: 109-110, 2010.

37. Fogel-Petrovic M, Vujcic S, Miller J and Porter CW: Differential post-transcriptional control of ornithine decarboxylase and spermidine-spermine $\mathrm{N}^{1}$-acetyltransferase by polyamines. FEBS Lett 391: 89-94, 1996.
38. Mastrantonio R, Cervelli M, Pietropaoli S, Mariottini P, Colasanti $M$ and Persichini T: HIV-Tat induces the Nrf2/ARE pathway through NMDA receptor-elicited spermine oxidase activation in human neuroblastoma cells. PLoS One 11: e0149802, 2016.

39. Pietropaoli S, Leonetti A, Cervetto C, Venturini A, Mastrantonio R, Baroli G, Persichini T, Colasanti M, Maura G, Marcoli M, et al: Glutamate Excitotoxicity Linked to Spermine Oxidase Overexpression. Mol Neurobiol 55: 7259-7270, 2018.

40. Caforio M, Sorino C, Iacovelli S, Fanciulli M, Locatelli F and Folgiero V: Recent advances in searching c-Myc transcriptional cofactors during tumorigenesis. J Exp Clin Cancer Res 37: 239, 2018.

41. Jing P, Zou J, Weng K and Peng P: The PI3K/AKT axis modulates AATF activity in Wilms' tumor cells. FEBS Open Bio 8: 1615-1623, 2018. 